Article

\title{
Uniformity Study of Two-Functional Luminescent Dyes Adsorbed over an Anodized Aluminum Coating for Motion-Capturing Pressure- and Temperature-Sensitive Paint Imaging
}

\author{
Masato Ishii ${ }^{1}$, Takeshi Miyazaki ${ }^{2}$ and Hirotaka Sakaue ${ }^{3, *}$ \\ 1 Mechanical Section, Second Forensic Science Division, National Research Institute of Police Science, \\ Kashiwa 277-0882, Japan; ishii_masato@nrips.go.jp \\ 2 Department of Mechanical Engineering and Intelligent System, University of Electro-Communications, \\ Chofu 182-8585, Japan; miyazaki@miyazaki.mce.uec.ac.jp \\ 3 Department of Aerospace and Mechanical Engineering, University of Notre Dame, Notre Dame, \\ IN 46556, USA \\ * Correspondence: hsakaue@nd.edu; Tel.: +1-574-631-4336
}

Received: 16 October 2017; Accepted: 20 December 2017; Published: 23 December 2017

\begin{abstract}
The pressure- and temperature-sensitive paint (PSP/TSP) technique, for steady-state and unsteady-state measurements, is becoming widespread. However, unsteady quantitative measurement is still difficult because non-uniform distribution of the probes over a test model may cause errors in the results. We focus on the dipping method that applies two luminophores into a binding material to improve sensitivity uniformity over a model surface. A bullet-shaped axisymmetric test model with motion-capturing TSP was used to evaluate the sensitivity uniformity, and three dipping methods (static, convectional, and rotational) were examined. The average peak ratios in the longitudinal direction were 1.17-1.46 for static, 1.38-1.51 for convectional, and 1.42-1.45 for rotational dipping. The standard deviations in the transverse direction were the smallest for rotational (0.022-0.033), relative to static (0.086-0.104), and convectional (0.044-0.065) dipping.
\end{abstract}

Keywords: sensitivity uniformity; PSP/TSP; unsteady measurement; dual luminescence

\section{Introduction}

The pressure- and temperature-sensitive paint (PSP/TSP) technique, for the steady-state and unsteady-state measurement of rotor blades, turbomachinery, wing fluttering, and aeroacoustic problems, is becoming widespread [1-11]. One of the unsteady measurement techniques is motion-capturing PSP/TSP, which utilizes luminescent output from two luminophores that have different pressure/temperature sensitivities [12]. In this method, one luminophore provides a signal and the other a reference output. The luminescence ratio distribution between a signal and a reference output on a model surface is converted to a pressure/temperature map by applying calibration data. Yamada et al. [13] and Ishii et al. [14] tried to use the motion-capturing PSP/TSP method to measure the surface pressure/temperature of a bullet-shaped axisymmetric object flying at the speed of sound. However, the pressure/temperature distribution was still aerodynamically incorrect.

Yamada et al. reported that the luminescence ratio pattern on a projectile surface would cause errors in surface temperature if a single set of calibration data was applied and the pattern had different local sensitivities. Thus, multiple calibration data sets are needed to determine local pressures and temperatures. However, it is difficult to apply multiple calibration data to a moving measurement model because it is not generally possible to fix an accurate position between calibration and experiment without complex image processing. Additionally, the existence of a luminescent pattern would be 
difficult to distinguish whether the change in luminescent output is based on a non-uniformity of a sensor sensitivity or caused by the physical phenomenon itself. Therefore, a uniformity of a sensor sensitivity over a measurement model needs to be improved. We focused on the dipping method to adsorb luminescent molecules over an axisymmetric test model, since an improvement of uniformity in sensitivity would advance the reliability of the quantitative PSP/TSP measurement.

In previous reports on dual luminescence methods [15-17], there were a few discussions about sensitivity uniformity, except for the dual-luminophore PSP/TSP of Mitsuo et al. [18]. They noted the importance of sensitivity uniformity of a model surface but did not evaluate it. Because the motion-capturing PSP/TSP method uses dual luminescence, the evaluation of the luminescence ratio distribution is necessary. Hence, the peak-ratio uniformity for two-color TSP was characterized here for two luminophores applied by a dipping deposition to a binding material.

\section{Materials and Methods}

\subsection{Preparation of Dipping Solutions}

Two-color TSP was used to evaluate the sensitivity uniformity over a model surface. Strong luminescent output was obtained with rhodamine B and fluorescein dyes as the signal and reference luminophores, respectively. They were separately dissolved in dichloromethane at concentrations of $0.005 \mathrm{mM}$ (signal) and $0.2 \mathrm{mM}$ (reference). The solutions were then mixed 1:1 by volume.

\subsection{Preparation of the Test Model}

Figure 1 shows the shape of the test model as well as the two axes used to compare the results of the dipping methods. The motion-capturing method requires anodization of an aluminum test model. The axes origin was at the top of the model, as shown in Figure 1, where the $X$-axis was along the center and the $Y$-axis was perpendicular to the $X$-axis.

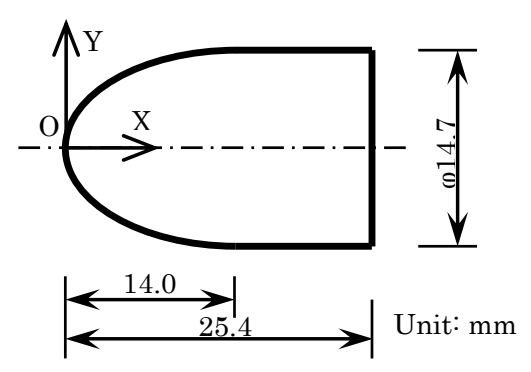

Figure 1. Shape of test model.

Figure 2 schematically depicts the anodization, which was generally based on Sakaue's procedure [19]. At first, the test model was rinsed in a $2 \mathrm{wt} \%$ sodium hydroxide solution for $5 \mathrm{~m}$ and washed by distilled water. After this procedure was repeated three times, the model was dehydrated by vacuum oven at room temperature. Then, the model was anodized by sulfuric acid with a concentration of $1 \mathrm{~mol} / \mathrm{L}$ and a temperature of $3{ }^{\circ} \mathrm{C}$ for $90 \mathrm{~m}$. The electric voltage was set at a $20 \mathrm{~V}$ constant during the anodization. After the anodization, the model was rinsed in a $3 \mathrm{wt} \%$ phosphoric acid solution for $20 \mathrm{~m}$ with a temperature of $30^{\circ} \mathrm{C}$. Again, the model was washed by distilled water and dehydrated by vacuum oven at room temperature. 


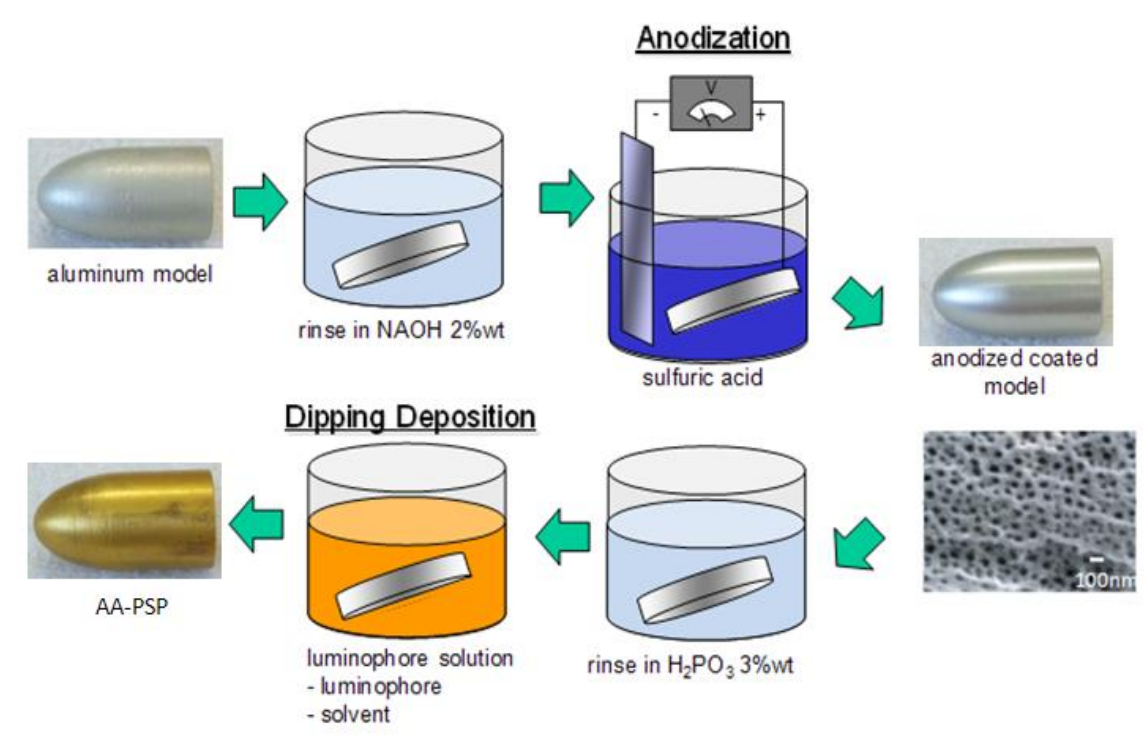

Figure 2. Schematic of the test model preparation.

\subsection{Dipping Methods}

The sensitivity uniformity was evaluated for three dipping methods depicted in Figure 3: static, convectional, and rotational dipping.

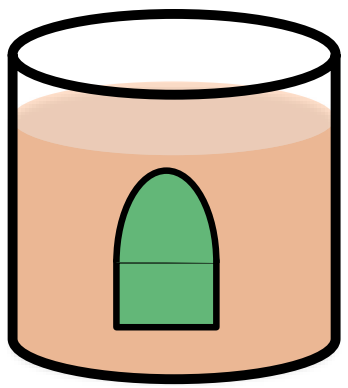

(a)

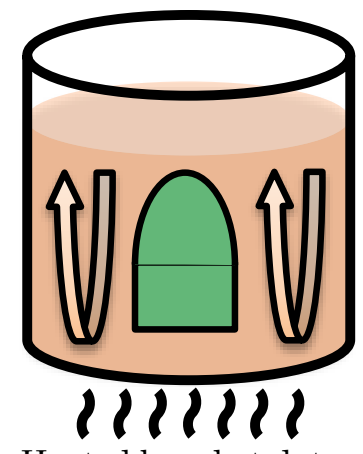

Heated by a hotplate

(b)

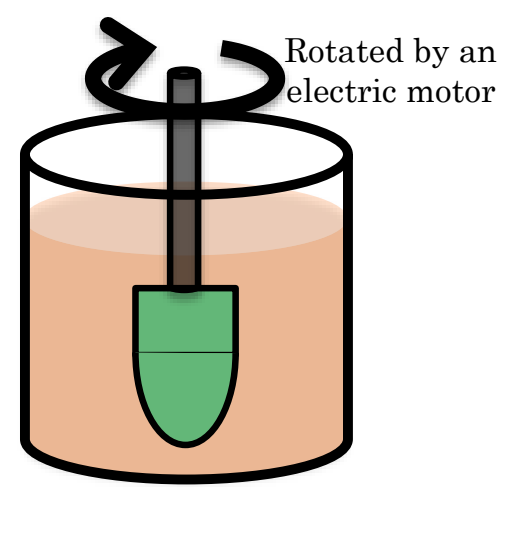

(c)

Figure 3. Schematic depictions of dipping methods. (a) Static dipping; (b) Convectional dipping; (c) Rotational dipping.

Static dipping, originally used for measurements of a free-flight object, involved dipping with a stationary solution and a stationary test model. The model rested for $5 \mathrm{~min}$ at the bottom of a beaker filled with a dipping solution that was not stirred.

Convectional dipping involved heating the solution at the bottom of the beaker during dipping. The solution was heated by a hotplate to a temperature of $35^{\circ} \mathrm{C}$. The stationary test model was then dipped into the solution and rested for $5 \mathrm{~min}$. Here, the solution temperature was kept at $35^{\circ} \mathrm{C}$ during dipping.

Rotational dipping involved rotation of the model by an electric motor and the rotational mechanism shown in Figure 4. The model was held vertically in the solution by a shaft with thread for $3 \mathrm{~min}$ at rotated at $870 \mathrm{rpm}$.

After each dipping procedure, the test model was dried in a vacuum oven at room temperature. 


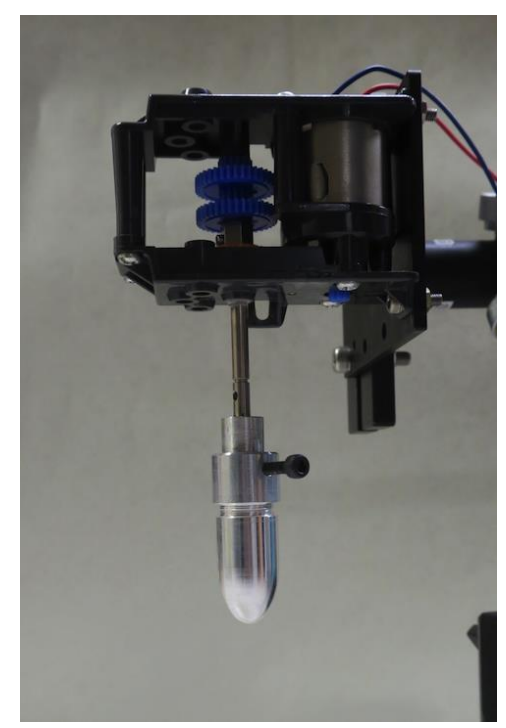

Figure 4. Rotational mechanism for rotational dipping.

\subsection{Luminescence Imaging}

The TSP-coated test model was put in the pressure chamber so that the luminescence image of the test model was acquired with a high-speed color camera (Phantom Miro M4, Vision Research Inc., Trenton, NJ, USA). The pressure and temperature of the pressure chamber had set at atmospheric pressure and room temperature. The camera exposure was set at $1.9 \mathrm{~ms}$ and the bit depth was 12 bits. The test model was illuminated by a $405 \mathrm{~nm}$ laser (BrixX 405-1200, Omicron-Laserage Laserprodukte $\mathrm{GmbH}$, Rodgau, Germany), whose output power was $1.2 \mathrm{~W}$ with $\mathrm{CW}$ mode, and the laser illumination was expanded to a $50 \mathrm{~mm}$ diameter by a collimator lens so that the signal and reference luminophores were excited simultaneously. A $422 \mathrm{~nm}$ high pass filter was placed in front of the camera lens to cut off the illumination luminescence. These setups gave a luminescence output from 0 to 4095 pixels. Figure 5 shows that the typical emission spectra of two-color TSP in this study consisted of rhodamine B and fluorescein excited by a $405 \mathrm{~nm}$ laser simultaneously. The peak of the spectra around $500 \mathrm{~nm}$ was the luminescence emission from fluorescein and the peak around $580 \mathrm{~nm}$ was from rhodamine $B$, respectively.

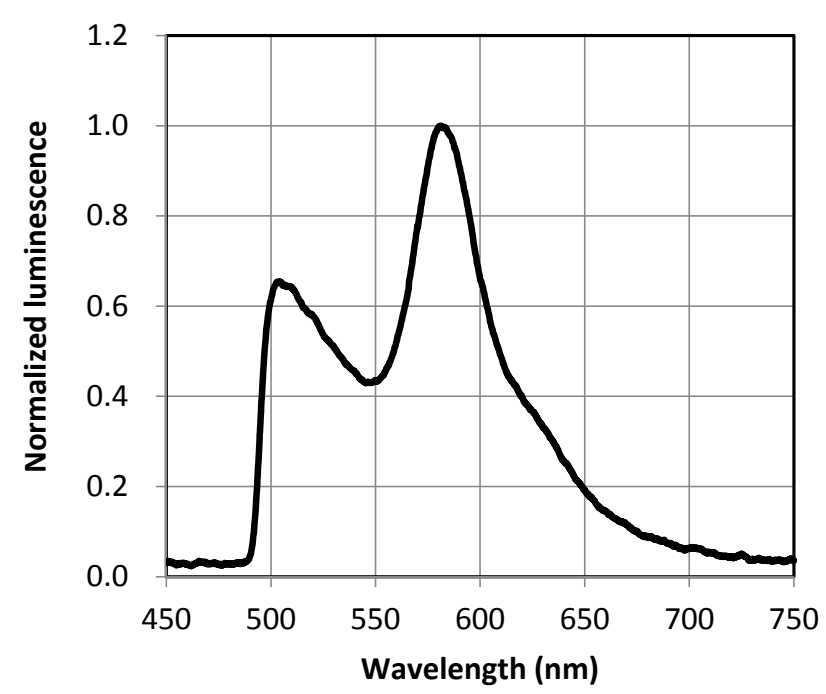

Figure 5. Emission spectra of two-color TSP consists of rhodamine B and fluorescein excited by a $405 \mathrm{~nm}$ laser. 
The luminescence images recorded on the image sensor were divided into red, green, and blue images. The red and green images were then used for measuring the luminescence ratio distributions. The luminescence ratio distribution was taken by simply divided the red image by the green image. All image processing was based on MATLAB 2015b (Mathworks, Inc., Boston, MA, USA).

\section{Results and Discussion}

Figure 6 shows the luminescence ratio distributions on the test model surfaces after the different dipping procedures. As shown by Figure $6 \mathrm{a}$, the ratio for the static method was highest at the top of the model and gradually decreased towards the bottom. The ratio obtained by the convectional method (Figure 6b) was highest at the bottom of the model. Finally, the ratio produced by the rotational method was almost constant across the whole surface, as shown in Figure 6c.

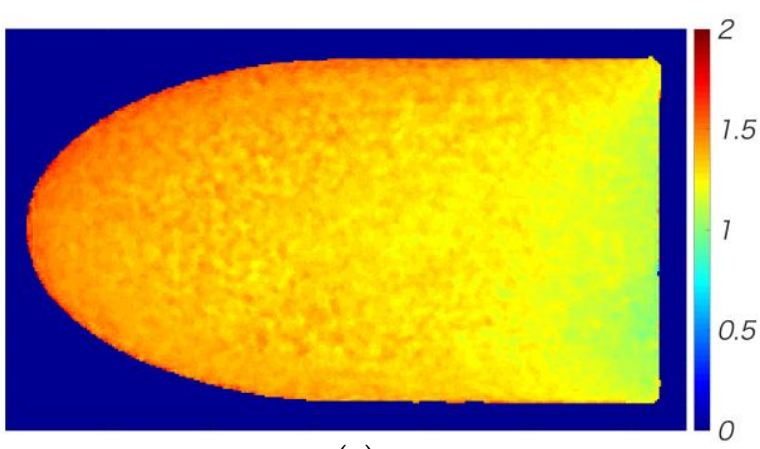

(a)

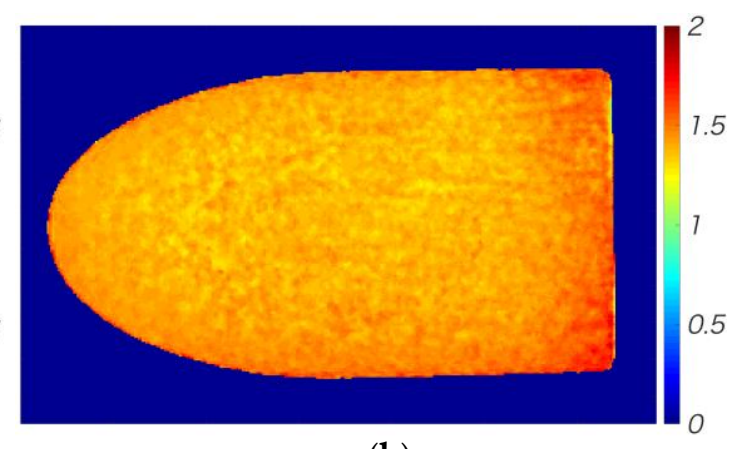

(b)

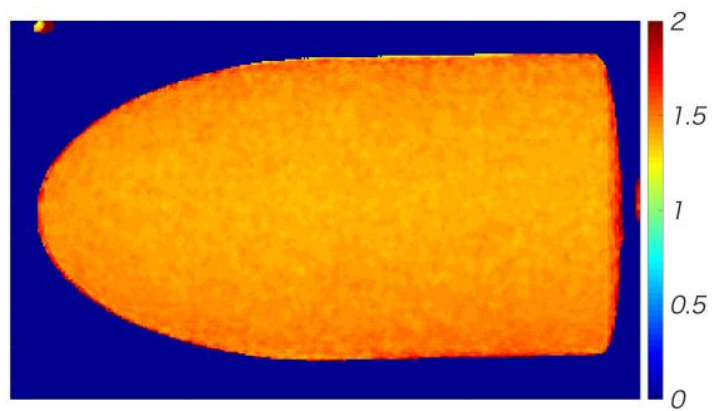

(c)

Figure 6. Luminescence ratio distributions on the test models. (a) Static dipping; (b) Convectional dipping; (c) Rotational dipping.

Based on the data in Figure 6, we evaluated the luminescence ratios in detail using the luminescent ratio profiles, and the position of the extraction is shown by the arrows in Figure 7 . Figure 8 plots the luminescent ratios along the $X$-axis (longitudinal direction) at $Y$ values of $-6,-3,0,3$, and $6 \mathrm{~mm}$. For static dipping, shown in Figure $8 \mathrm{a}$, the ratios were highest at the top of the model and gradually lower towards the bottom, regardless of the Y-positions. The convectional data in Figure $8 \mathrm{~b}$ indicates that the ratios were almost uniform along the model surface, except near the bottom where it increased. Finally, the plots of rotational data in Figure $8 \mathrm{c}$ indicate almost uniform ratios along the whole model surface. 


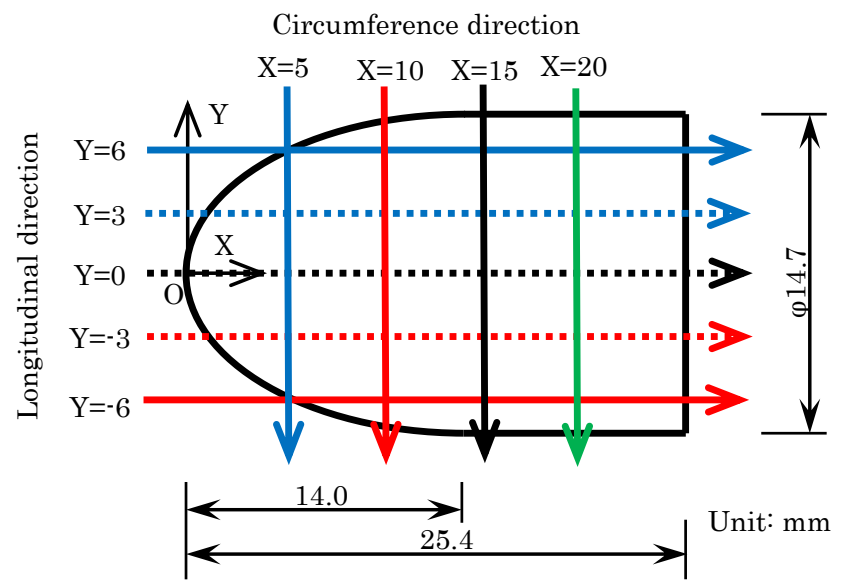

Figure 7. Position of the luminescence ratio profile extraction.

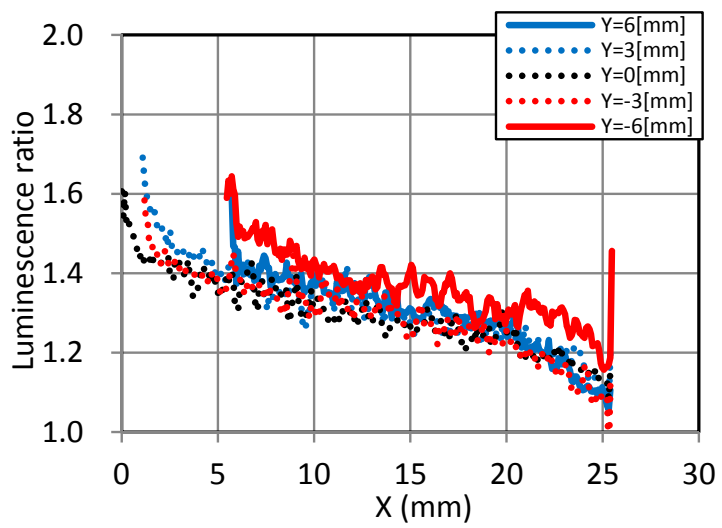

(a)

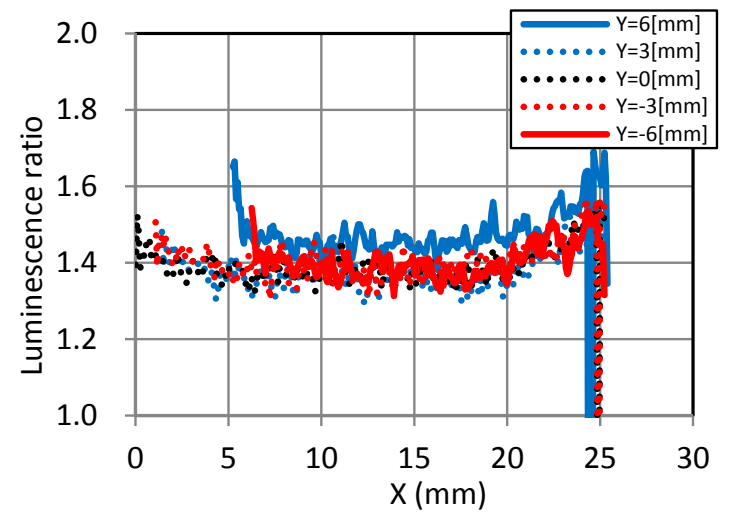

(b)

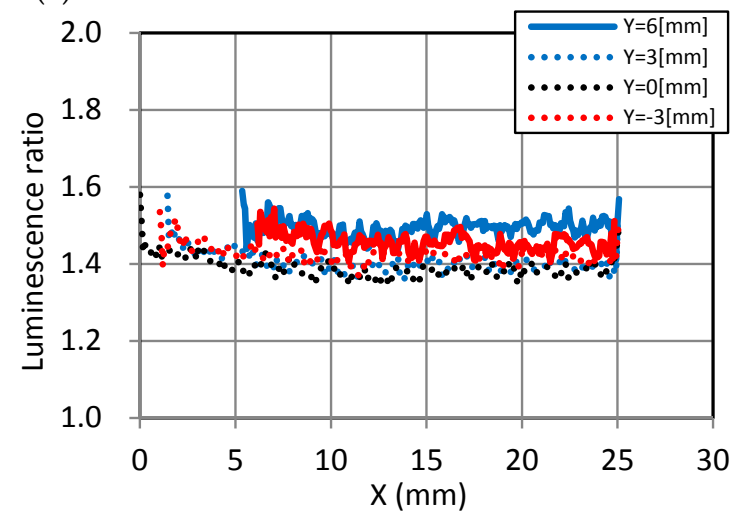

(c)

Figure 8. Luminescence ratios along the $X$-axis (longitudinal direction).

(a) Static dipping;

(b) Convectional dipping; (c) Rotational dipping.

Figure 9 shows the statistical plots of the luminescence ratio distributions along the $X$-axis. These lines indicate the averages and the standard deviations of luminescence ratios in the circumference direction over each test models. In Figure 9a, the averaged luminescence ratios for the static dipping method gradually changes from 1.46 at the top of the model to 1.17 at the bottom. In the convectional data (Figure $9 \mathrm{~b}$ ), it changes from 1.38 at the top to 1.51 near the bottom. The rotational data are all within 1.42-1.45, clearly indicating that this dipping method is the most uniform. The standard deviation in the longitudinal direction was similar for all three dipping procedures, as shown in 
Figure 9b. According to the plot in Figure 9b, there was almost no difference of uniformity in sensitivity between the dipping methods in the circumference direction.

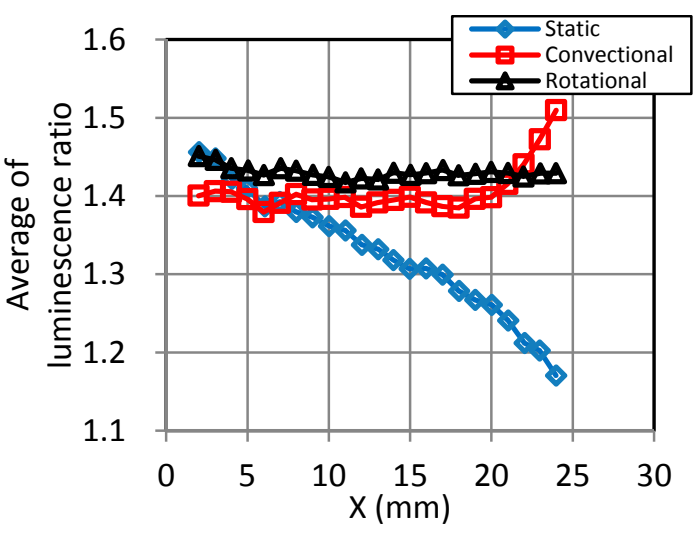

(a)

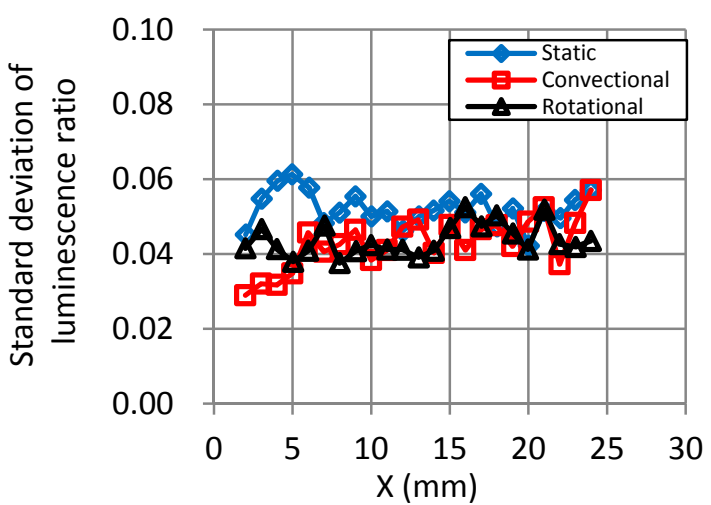

(b)

Figure 9. Statistical plots of luminescence ratios along the $X$-axis. (a) Average; (b) Standard deviation.

Figure 10 shows the luminescence ratio plots along the $Y$-axis (circumference direction) at $X$ values of 5, 10, 15, and $20 \mathrm{~mm}$. For the static dipping data, shown in Figure 10a, the ratios decreased with increasing $X$ values. The convectional data in Figure $10 \mathrm{~b}$ and the rotational data in Figure $10 \mathrm{c}$ were almost uniform along with the model surface.

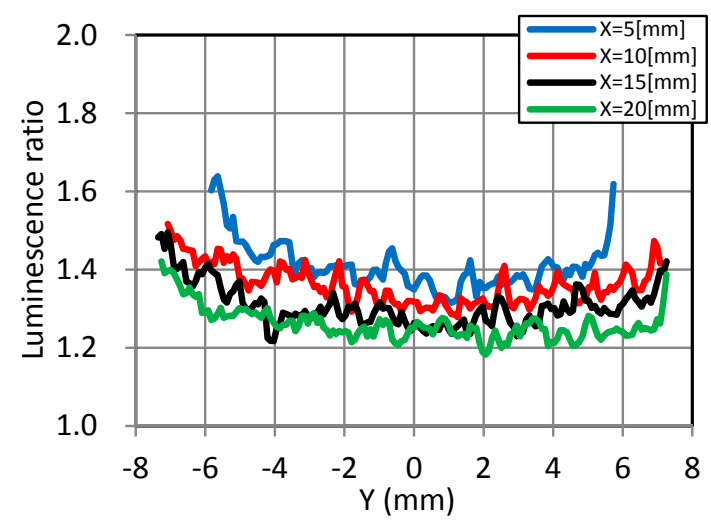

(a)

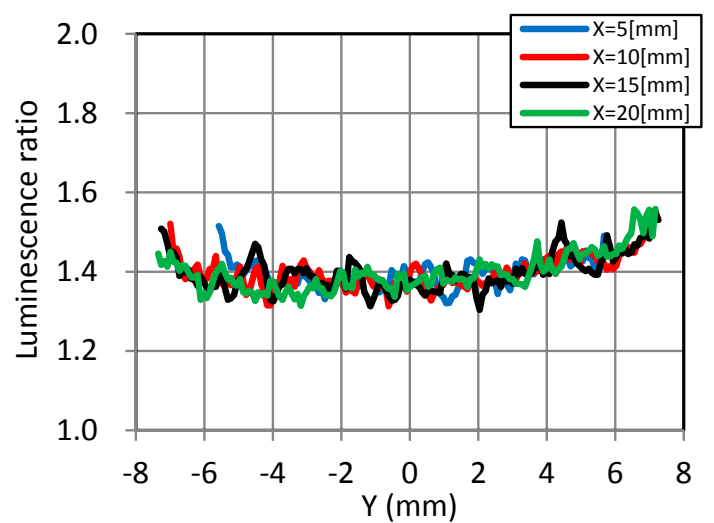

(b)

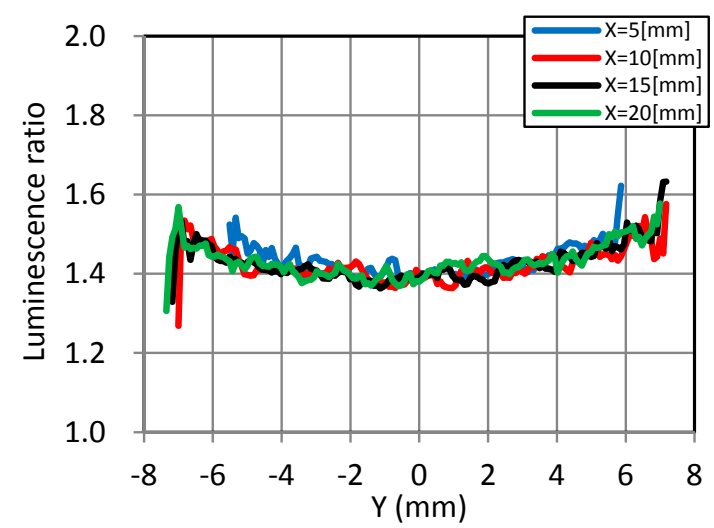

(c)

Figure 10. Luminescence ratio plots along the $Y$-axis (circumference direction). (a) Static dipping; (b) Convectional dipping; (c) Rotational dipping. 
Figure 11 shows the statistical plots of the luminescence ratios along the $Y$-axis. These lines indicate the averages and the standard deviations of luminescence ratios in the longitudinal direction over each test models. Figure 11a indicates that the averaged luminescence ratios obtained with convectional dipping was 1.38-1.48, and those for rotational dipping were similar at 1.39-1.49. In addition, the plots were symmetrical, about $Y=0$, because of the configuration of the axisymmetric measurement model. Finally, the averaged ratios for the static method were 1.29-1.37, and these are lower than the others. Figure $11 \mathrm{~b}$ reveals that the standard deviations for the static, convectional, and rotational dipping methods were 0.086-0.104, 0.044-0.065, and 0.022-0.033, respectively. Hence, the plot in Figure $11 \mathrm{~b}$ indicates that the standard deviation in the longitudinal direction was lowest for the rotational method and highest for the static method, so the uniformity in sensitivity improved more so by the former method.

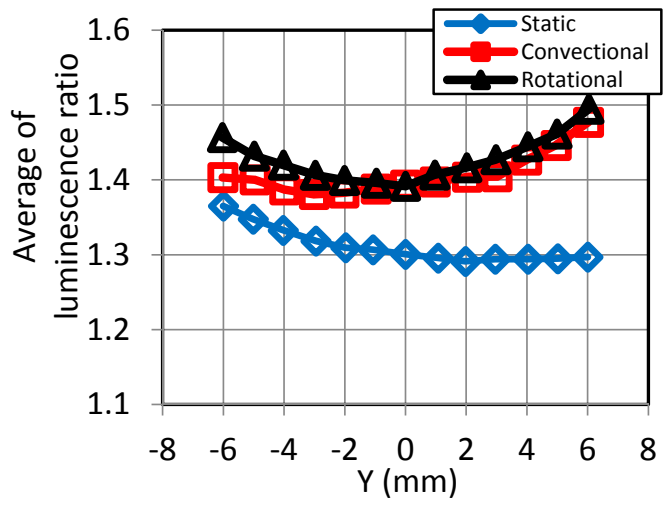

(a)

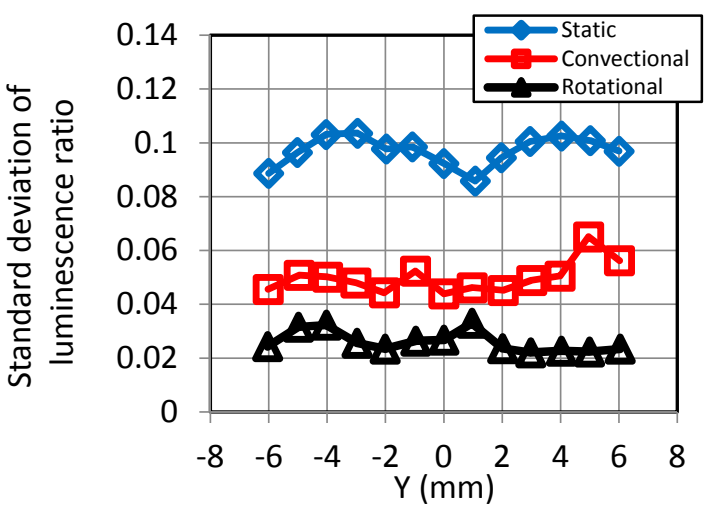

(b)

Figure 11. Statistical plots of luminescence ratios along the $Y$-axis. (a) Average; (b) Standard deviation.

Based on these results above, both the convectional and rotational methods, compared to the static method, showed better uniformity in sensitivity. This means that the dipping solution movement affected the luminophore distribution on the axisymmetric test model. Additionally, the rotational method improved uniformity more so than the convectional method. The difference between these two methods is caused by the convection types of the solution. The convection type is classified as natural in the convectional method and as forced in the rotational method. Natural convection generally provides a vertical solution movement, and forced convection provides movement in the vertical and circumference directions. This means that the movement direction of the model in the solution influenced the luminophore distribution. It can be concluded, therefore, that the rotational method produced the most uniform luminescence ratio distribution on the surface of the axisymmetric measurement model. These results showed that adopting the rotational dipping method would solve the non-uniformity of luminophores on an axisymmetric test model such that the effect of error caused by different local sensitivities is eliminated. The improvement of sensitivity uniformity will advance the reliability of quantitative PSP/TSP measurement.

\section{Conclusions}

We evaluated the peak-ratio uniformity of a two-color TSP using an axisymmetric measurement model. Three dipping deposition methods were tested to apply two luminophores onto binding materials. The range of averaged luminescence ratios in the longitudinal direction was 1.17-1.46 for static dipping, 1.38-1.51 for convectional dipping, and 1.42-1.45 for rotational dipping. The averaged ratios in the circumference direction were 1.29-1.37 for static, 1.38-1.48 for convectional, and 1.39-1.49 for rotational dipping. However, the ratio distribution obtained from the static dipping was different from the others. Finally, the range of standard deviation in the transverse direction was smallest for 
the rotational method $(0.022-0.033)$, compared with those for the static $(0.086-0.104)$ and convectional (0.044-0.065) methods.

Acknowledgments: The authors would like to thank Steven Claucherty at University of Notre Dame for his technical support.

Author Contributions: H.S. and T.M. conceived and designed the experiments; H.S. performed the experiments; M.I., T.M., and H.S. analyzed the data; H.S. contributed reagents/materials/analysis tools; M.I. wrote the paper.

Conflicts of Interest: The authors declare no conflict of interest.

\section{References}

1. Bell, J.H.; Schairer, E.T.; Hand, L.A.; Mehta, R.D. Surface pressure measurements using luminescent coatings. Annu. Rev. Fluid Mech. 2001, 33, 155-206. [CrossRef]

2. Gregory, J.W.; Sakaue, H.; Liu, T.; Sullivan, J.P. Fast pressure sensitive pant for flow and acoustics diagnostics. Annu. Rev. Fluid Mech. 2014, 46, 303-330. [CrossRef]

3. Disotell, K.J.; Peng, D.; Juliano, T.J.; Gregory, J.W.; Crafton, J.W.; Komerath, N.M. Single-shot temperatureand pressure-sensitive paint measurements on an unsteady helicopter blade. Exp. Fluids 2014, 55, 1671. [CrossRef]

4. Gregory, J.W. Porous pressure-sensitive paint for measurement of unsteady pressure in turbomachinery. In Proceedings of the 42nd AIAA Aerospace Sciences Meeting and Exhibit, Aerospace Sciences Meetings, Reno, NV, USA, 5-8 January 2004. [CrossRef]

5. Gregory, J.W.; Sullivan, J.P.; Wannis, S.S.; Komerath, N.M. Pressure-sensitive paint as a distributed optical microphone array. J. Acoust. Soc. Am. 2006, 119, 251-261. [CrossRef] [PubMed]

6. Nakakita, K. Unsteady pressure distribution measurement around 2D-cylinders using pressure-sensitive paint. In Proceedings of the 25th AIAA Applied Aerodynamics Conference, Fluid Dynamics and Co-Located Conferences, Miami, FL, USA, 25-28 June 2007. [CrossRef]

7. Nakakita, K. Unsteady pressure measurement on NACA0012 model using global low-speed unsteady PSP technique. In Proceedings of the 41st AIAA Fluid Dynamics Conference and Exhibit, Fluid Dynamics and Co-Located Conferences, Honolulu, HI, USA, 27-30 June 2011. [CrossRef]

8. Okabe, T.; Miyazaki, T.; Sakaue, H.; Saitoh, K. Motion-Capturing PSP system for flutter application. In Proceedings of the 41st AIAA Fluid Dynamics Conference and Exhibit, Fluid Dynamics and Co-Located Conferences, Honolulu, HI, USA, 27-30 June 2011. [CrossRef]

9. Okabe, T.; Miyazaki, T.; Saitoh, K.; Sakaue, H. Unsteady pressure distribution on a fluttering airfoil using Motion-Capturing PSP system. In Proceedings of the 44th AIAA Thermophysics Conference, Fluid Dynamics and Co-Located Conferences, San Diego, CA, USA, 24-27 June 2013. [CrossRef]

10. Sakaue, H.; Iijima, Y.; Yamada, Y.; Miyazaki, T.; Ishii, M. Global pressure and temperature measurements of ballistic-range testing by PSP and TSP technique. In Proceedings of the 52nd Aerospace Sciences Meeting, AIAA SciTech Forum, National Harbor, MD, USA, 13-17 January 2014. [CrossRef]

11. Hird, K.; Juliano, T.J.; Gregory, J.W. Study of unsteady surface pressure on a turret via pressure-sensitive paint. In Proceedings of the 44th AIAA Thermophysics Conference, Fluid Dynamics and Co-Located Conferences, San Diego, CA, USA, 24-27 June 2013. [CrossRef]

12. Sakaue, H.; Miyamoto, K.; Miyazaki, T. A motion-capturing pressure-sensitive paint method. J. Appl. Phys. 2013, 113, 084901. [CrossRef]

13. Yamada, Y.; Ishii, M.; Miyazaki, T.; Sakaue, H. Global pressure measurement of a moving bullet. In Proceedings of the 44th AIAA Thermophysics Conference, Fluid Dynamics and Co-Located Conferences, San Diego, CA, USA, 24-27 June 2013. [CrossRef]

14. Ishii, M.; Isokawa, H.; Miyazaki, T.; Sakaue, H. Surface state measurement of a free-flight object by motion-capturing method. In Proceedings of the 55th AIAA Aerospace Sciences Meeting, AIAA SciTech Forum, Grapevine, TX, USA, 9-13 January 2017. [CrossRef]

15. Iijima, Y.; Sakaue, H. Platinum porphyrin and luminescent polymer for two-color pressure- and temperature-sensing probe. Sens. Actuators A Phys. 2012, 184, 128-133. [CrossRef] 
16. Khalil, G.E.; Costin, C.; Crafton, J.; Jones, G.; Grenoble, S.; Gouterman, M.; Callis, J.B.; Dalton, L.R. Dual luminophor pressure sensitive paint I. Ratio of reference to sensor giving a small temperature dependency. Sens. Actuators B Chem. 2004, 97, 13-21. [CrossRef]

17. Zelelow, B.; Khalil, G.E.; Phelen, G.; Carlson, B.; Gouterman, M.; Callis, J.B.; Dalton, L.R. Dual luminophor pressure sensitive paint II. Lifetime based measurement of pressure and temperature. Sens. Actuators B Chem. 2003, 96, 304-314. [CrossRef]

18. Mitsuo, K.; Asai, K.; Hayasaka, M.; Kameda, M. Temperature correction of PSP measurement using dual-luminophor coating. J. Vis. 2003, 6, 213-223. [CrossRef]

19. Sakaue, H. Luminophore application method of anodized aluminum pressure sensitive paint as a fast responding global pressure sensor. Rev. Sci. Instrum. 2005, 76, 084101. [CrossRef]

2017 by the authors. Licensee MDPI, Basel, Switzerland. This article is an open access article distributed under the terms and conditions of the Creative Commons Attribution (CC BY) license (http:// creativecommons.org/licenses/by/4.0/). 